\title{
Identification of the SGR6065 gene product as a sesquiterpene cyclase involved in ( + )-epicubenol biosynthesis in Streptomyces griseus
}

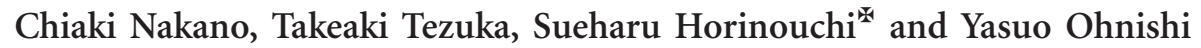

Recent bacterial genome sequencing projects have shown the presence of many putative sesquiterpene cyclase (SC) genes, especially in the Gram-positive, filamentous bacterial genus Streptomyces. We describe here the characterization of a SC gene (SGR6065, named gecA) from Streptomyces griseus. Overexpression of gecA in Streptomyces lividans produced a sesquiterpene, which was isolated and determined to be $(+)$-epicubenol using spectroscopic analyses. The $\mathrm{N}$-terminal histidine-tagged GecA protein was produced in Escherichia coli. Incubation of the recombinant GecA protein with farnesyl diphosphate (FPP) yielded (+)-epicubenol as the major product. The $K_{\mathrm{m}}$ value for FPP and the $\boldsymbol{k}_{\text {cat }}$ value for $(+)$-epicubenol formation were calculated to be $254 \pm 7.1 \mathrm{~nm}$ and $0.026 \pm 0.001 \mathrm{~s}^{-1}$, respectively. The $k_{\text {cat }} / K_{\mathrm{m}}$ value $\left(0.10 \mathrm{~s}^{-1} \mu \mathrm{m}^{-1}\right)$ was broadly comparable to those reported for known bacterial SCs. ( + )-Epicubenol was detected in the crude cell lysate of wild-type S. griseus, but not in a gecA-knockout mutant, indicating that GecA is a genuine (+)-epicubenol synthase. Although (+)-epicubenol synthases have been previously purified and characterized from the liverwort Heteroscyphus planus and Streptomyces sp. LL-B7, no ( + )-epicubenol synthase gene has been cloned to date. The gecA gene is thus the first example of an (+)-epicubenol synthase-encoding gene. $(+)$-Epicubenol production was not controlled by the microbial hormone A-factor that induces morphological differentiation and production of several secondary metabolites in S. griseus.

The Journal of Antibiotics (2012) 65, 551-558; doi:10.1038/ja.2012.68; published online 8 August 2012

Keywords: (+ )-epicubenol; genome mining; natural product; sesquiterpene cyclase; Streptomyces griseus

\section{INTRODUCTION}

Terpenoids are one of the major classes of natural products and have various biological roles, including as flavorings, pigments, antibiotics and hormones. ${ }^{1}$ They are biosynthesized from the $\mathrm{C}_{5}$ precursors isopentenyl diphosphate and dimethylallyl diphosphate. In the biosynthesis of terpenoids, terpenoid cyclases are key enzymes in all pathways leading to the diverse range of terpenoids. Terpenoid cyclases catalyze the cyclization of linear isoprenyl diphosphate, which is produced by the successive condensation of isopentenyl diphosphate and dimethylallyl diphosphate, to produce the parent skeletons of terpenoids. In contrast to the large number of terpenoid compounds produced by plants and fungi, bacteria produce only a small number of terpenoids. However, recent genome sequencing projects have revealed many potential bacterial terpenoid cyclase genes, especially sesquiterpene cyclase (SC) genes in Streptomyces. $\mathrm{SCs}$ catalyze the cyclization of farnesyl diphosphate (FPP; $\mathrm{C}_{15}$ ) into any of 300 known hydrocarbon skeletons. Using a genome mining approach, several bacterial SC homologs have been identified from Streptomyces and Nostoc species. These proteins are involved in the synthesis of germacradienol/geosmin, ${ }^{2-5}$ pentalenene, ${ }^{6}$ epi-isozizaene, ${ }^{7,8}$ avermitilol, ${ }^{9}$ germacrene $A,{ }^{10}$ 8a-epi- $\alpha$-selinene, ${ }^{10}$ $(-)-\delta$-cadinene ${ }^{11}$ and $(+)$-T-muurolol. ${ }^{11}$ Germacradienol/ geosmin synthase is a representative bacterial SC, but is atypical in that it consists of two terpene cyclase domains. Others are typical single-domain SCs. Recently, we reported the identification and characterization of five bacterial single-domain SC homologs: the $(+)$-caryolan-1-ol synthase $\mathrm{GcoA}^{12}$ in Streptomyces griseus, the 1,8-cineole synthase $\mathrm{CnsA}^{13}$ and linalool/nerolidol synthase LnsA ${ }^{14}$ in Streptomyces clavuligerus, and the (-)-germacradien-4-ol synthase $S C 1^{15}$ and (-)-epi- $\alpha$-bisabolol synthase SC2 ${ }^{15}$ in Streptomyces citricolor. CnsA is the first bacterial monoterpene synthase known to catalyze the direct conversion of geranyl diphosphate, ${ }^{13}$ whereas LnsA is the first bacterial acyclic terpene synthase that shows significant amino-acid sequence similarity to bacterial SCs. ${ }^{14}$ These studies and the presence of a large number of terpene cyclase homologs in bacteria indicate that terpenoids are likely to be significantly more widely distributed in nature than previously appreciated. ${ }^{16}$ 
The bicyclic sesquiterpene alcohol $(+)$-epicubenol has been isolated previously not only from plants, ${ }^{17,18}$ but also from three Streptomyces species. ${ }^{19}$ The enzymatic conversion of FPP to $(+)$-epicubenol has been previously performed using a purified enzyme, as well as a crude extract, prepared from the liverwort Heteroscyphus planus ${ }^{20,21}$ and Streptomyces sp. LL-B7. ${ }^{22-25}$ Cane et al. have presented extensive experimental evidence for a cyclization mechanism of the $(+)$-epicubenol synthase from Streptomyces sp. LLB7 (Figure 1). The authors demonstrated that the reaction is initiated by isomerization of FPP to $(3 R)$-nerolidyl diphosphate, which then undergoes conversion to the intermediate $(Z, E)$-germacradienyl cation, 2. After a 1,3-hydride shift and a second cyclization, the cis-fused muurolyl cation, 3, is generated, and then a 1,2-hydride shift occurs to give the bicyclic cation, 4. Finally, this cation is quenched by the addition of a water molecule to produce (+)-epicubenol. ${ }^{22-25}$ Nabeta et al. ${ }^{20}$ demonstrated the same reaction mechanism using cell-free extracts of cultured cells of $H$. planus. To date, however, no gene encoding an $(+)$-epicubenol synthase has been cloned from any organism.

In 2008, we reported the complete genome sequence of $S$. griseus IFO13350. ${ }^{26}$ We have long studied the A-factor regulatory cascade that leads to secondary metabolism and morphological differentiation in this famous streptomycin producer. ${ }^{27}$ A-factor (2-isocapryloyl-3Rhydroxymethyl- $\gamma$-butyrolactone) is produced by the action of AfsA, which is the key enzyme for A-factor biosynthesis, and is gradually accumulates in a growth-dependent manner. ${ }^{28}$ When the A-factor concentration reaches a critical level, A-factor binds the A-factorspecific receptor, which has bound and repressed the promoter of $a d p A$, and dissociates A-factor-specific receptor from the promoter, resulting in induction of $a d p A$ transcription. ${ }^{29}$ The AraC/XylS family transcriptional regulator AdpA then activates a number of genes required for morphological differentiation and secondary metabolism, forming an AdpA regulon. ${ }^{30,31}$ We identified two single-domain SC homologs (SGR2079 and SGR6065) in the complete genome of S. griseus. Last year, we demonstrated that SGR2079 (named GcoA) is the first SC known to synthesize (+)-caryolan-1-ol, and that the production of this secondary metabolite is under the control of A-factor. ${ }^{12}$ AdpA appeared to directly activate transcription of $g c o A$. In contrast, DNA microarray analysis predicted that transcription of the SGR6065 gene is independent of AdpA. ${ }^{26}$ SGR6065 (358 amino acids) shows $28 \%$ amino-acid sequence identity with the pentalenene synthase from Streptomyces sp. UC5319, ${ }^{32}$ and has both the aspartaterich motif (DDQLD, from Asp81 to Asp85) and the NSE/DTE motif (NDVYSFEKE, from Asn226 to Glu234), which are responsible for the $\mathrm{Mg}^{2+}$-diphosphate-enzyme complex formation, as well as the ionization of the substrate. ${ }^{33}$ Here we describe the characterization of the function of SGR6065 in vivo and in vitro. We reveal that SGR6065 is an $(+)$-epicubenol synthase, and have named the SGR6065 gene 'gecA' (S. griseus epicubenol synthase). (+ )-Epicubenol was detected in the crude cell lysate of wild-type $S$. griseus and an adpA-deleted mutant, but not in a gecA-knockout mutant, indicating that GecA is a genuine $(+)$-epicubenol synthase and that $(+)$-epicubenol production is not controlled by A-factor in S. griseus.

\section{MATERIALS AND METHODS}

\section{General analytical methods}

NMR spectra were recorded in $\mathrm{C}_{6} \mathrm{D}_{6}$ on a JNM-A500 spectrometer (JEOL, Tokyo, Japan). Chemical shifts in ${ }^{1} \mathrm{H}-$ and ${ }^{13} \mathrm{C}-\mathrm{NMR}$ spectra (p.p.m.) were relative to 7.15 and 128.0 p.p.m. for the solvent peak of $\mathrm{C}_{6} \mathrm{D}_{6}$ solution. The coupling constant $J$ was given in Hz. GC-MS analysis was performed using a JEOL JMS-Q1000 GC K9 instrument under electronic impact at $70 \mathrm{eV}$ with an Intercap 5MS/Sil capillary column $(15 \mathrm{~m} \times 0.25 \mathrm{~mm}$ ID, $0.25 \mu \mathrm{m}$ film thickness, GL science, Tokyo, Japan), the oven temperature being elevated from 60 (3-min hold) to $260^{\circ} \mathrm{C}\left(20^{\circ} \mathrm{C} \mathrm{min}-1\right)$. The specific rotation value was measured with a JASCO DIP-1000 digital polarimeter (Jasco, Tokyo, Japan) at room temperature.

\section{Bacterial strains, plasmids, media and chemicals}

Escherichia coli strains JM109 and BL21(DE3), plasmids pUC19 and pColdI, restriction enzymes and other DNA-modifying enzymes used for DNA<smiles>CCCCC=C(C)CCC=C(C)CCC=C(C)C</smiles>

FPP<smiles>C=C[C@](C)(CC/C=C(\C)CCC=C(C)C)OP</smiles>

(3R)-NPP

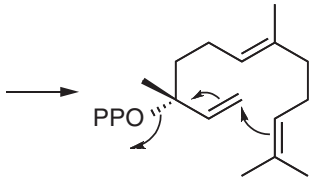

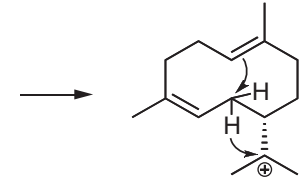

$(Z, E)$-germacradienyl cation (2)

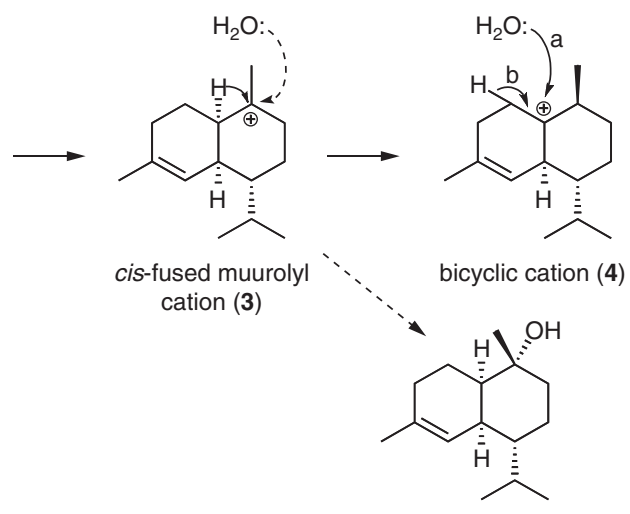

(+)-T-muurolol

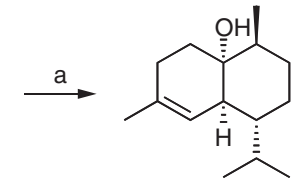

(+)-epicubenol (1)

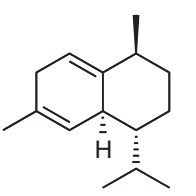

(+)-cubenene

Figure 1 Reactions proposed to be catalyzed by (+)-epicubenol synthase. (+)-Epicubenol synthase first catalyzes the isomerization of FPP to (3R)nerolidyl diphosphate and its subsequent ionization-initiated cyclization to yield ( $Z, E$ )-germacradienyl cation (2). It then catalyzes a 1,3-hydride shift and a second cyclization to give the cis-fused muurolyl cation (3). A 1,2-hydride shift then occurs to form the bicyclic cation (4), 4 is then quenched by the addition of a water molecule to produce $(+)$-epicubenol (path a). (+)-Cubenene is produced by deprotonation of 4 (path b). The structure of $(+)$-T-muurolol, which is produced by the addition of a water molecule to the cis-fused muurolyl cation (3), is also shown. 
manipulations were purchased from Takara Bio (Otsu, Japan). S. griseus IFO13350 was obtained from the Institute of Fermentation, Osaka, Japan (IFO). S. lividans TK21 was obtained from David A. Hopwood (John Innes Centre, Norwich, UK). Plasmid pHSA81, used for heterologous expression of gecA in S. lividans, was obtained from Michihiko Kobayashi (University of Tsukuba, Ibaraki, Japan). S. lividans and S. griseus were cultured in yeast extract-malt extract (YEME) medium (glucose, 1\%; sucrose, 34\%; yeast extract, $0.3 \%$; malt extract, $0.3 \%$; bacto peptone, $0.5 \% ; \mathrm{MgCl}_{2} \cdot 6 \mathrm{H}_{2} \mathrm{O}, 0.1 \%$; $\mathrm{pH}$ 7.0) and YMPD medium, ${ }^{26}$ respectively. FPP was purchased from SigmaAldrich (St Louis, MO, USA). Authentic ( - )-germacradien-4-ol was produced by the in vitro reaction of recombinant $\mathrm{SC} 1$ with $\mathrm{FPP}^{15}$

\section{Construction of plasmids}

Chromosomal DNA of S. griseus was used as a template for the PCR amplification of gecA with primer I $5^{\prime}$-CCGAATTCCATATGGACAGC GAACTGCCGGACATCTAC-3' (EcoRI site underlined, NdeI site italicized and the start codon of gecA shown in boldface) and primer II $5^{\prime}$-CCGAAG CTTGGATCCTCAGCGGGCCCGGGGAGCCTCG-3' (HindIII site underlined and BamHI site italicized). The PCR product was digested with EcoRI and HindIII, and cloned between the EcoRI and HindIII sites of pUC19. The DNA fragments amplified by PCR were sequenced to confirm that no PCR error was introduced. The resultant plasmid pUC19-gecA was digested with NdeI and BamHI, and cloned between the NdeI and BamHI sites of pColdI and pHSA81, resulting in pColdI-gecA and pHSA81-gecA, respectively.

Analysis of terpenoids produced by recombinant S. lividans strains S. lividans harboring pHSA81-gecA (or an empty vector pHSA81) was cultivated at $30^{\circ} \mathrm{C}$ for $72 \mathrm{~h}$ in YEME medium $(100 \mathrm{ml})$ supplemented with $5 \mu \mathrm{g} \mathrm{ml}^{-1}$ thiostrepton. Cells were harvested by centrifugation and resuspended in $50 \mathrm{~mm}$ Tris- $\mathrm{HCl}(\mathrm{pH} 8.0,20 \mathrm{ml})$, then disrupted by sonication. The low-molecular-weight molecules were then extracted with ethyl acetate from the crude cell lysate. The organic layer was dried with $\mathrm{Na}_{2} \mathrm{SO}_{4}$, and evaporated to dryness. The residue was dissolved in ethyl acetate $(5 \mathrm{ml})$ for GC-MS analysis.

\section{Large-scale preparation and isolation of sesquiterpene}

S. lividans harboring pHSA81-gecA was cultivated at $30^{\circ} \mathrm{C}$ for $96 \mathrm{~h}$ in YEME medium $(4 \times 11)$ supplemented with $5 \mu \mathrm{g} \mathrm{ml}^{-1}$ thiostrepton. Cells were harvested by centrifugation and resuspended in $50 \mathrm{~mm}$ Tris- $\mathrm{HCl}(\mathrm{pH} 8.0$, $500 \mathrm{ml}$ ), then disrupted by sonication. Low-molecular-weight molecules were then extracted with ethyl acetate from the crude cell lysate. The organic layer was dried with $\mathrm{Na}_{2} \mathrm{SO}_{4}$ and evaporated to dryness. The crude materials were dissolved in a small amount of $n$-hexane. Sesquiterpene produced was purified by $\mathrm{SiO}_{2}$ column chromatography with $n$-hexane:ethyl acetate (100:0 to 100:5), yielding $9.2 \mathrm{mg}$ of $\mathbf{1}$. The structure of $\mathbf{1}$ was determined by NMR using ${ }^{1} \mathrm{H}$, ${ }^{13} \mathrm{C}$, DEPT, ${ }^{1} \mathrm{H}_{-}{ }^{1} \mathrm{H}$ COSY, NOESY, HMQC and HMBC experiments, and specific rotation analysis (Supplementary Figure S1).

\section{Production and purification of the recombinant GecA protein}

To produce N-terminal $\mathrm{His}_{6}$-tagged fusion proteins of GecA, E. coli BL21(DE3) strain harboring pColdI-gecA was grown at $37^{\circ} \mathrm{C}$ in Luria-Bertani medium $(200 \mathrm{ml})$ supplemented with $50 \mu \mathrm{g} \mathrm{ml}^{-1}$ ampicillin. When the $\mathrm{OD}_{600}$ had reached 0.4 , the culture was kept at $15^{\circ} \mathrm{C}$ for $30 \mathrm{~min}$. isopropylthiogalactoside (final $10 \mu \mathrm{M}$ ) was added and cultivation was continued for $24 \mathrm{~h}$ at $15^{\circ} \mathrm{C}$. Cells were harvested by centrifugation and resuspended in buffer A $(50 \mathrm{~mm}$ Tris$\mathrm{HCl}, 150 \mathrm{~mm} \mathrm{NaCl}, 5 \mathrm{~mm}$ imidazole and $10 \%$ glycerol, $\mathrm{pH} 8.0,40 \mathrm{ml})$. After sonication, cell debris was removed by centrifugation at $10000 \mathrm{~g}$ for $20 \mathrm{~min}$. Recombinant His-tagged protein was purified over a nickel-nitrilotriacetic acid superflow (Qiagen, Hilden, Germany) according to the manufacturer's instructions, with the exception that $10 \%$ glycerol was added to all buffers. The purified GecA protein was dialyzed against buffer B $(10 \mathrm{~mm}$ Tris- $\mathrm{HCl}$ and $10 \%$ glycerol, $\mathrm{pH} 8.0$ ). The purity of the recombinant proteins was checked by SDS-polyacrylamide gel electrophoresis. The protein concentration was measured with a NanoDrop 2000 spectrophotometer (Thermo Scientific, Wilmington, DE, USA).

\section{In vitro enzyme assay}

For the analysis of sesquiterpenes, the reaction mixture consisted of $50 \mathrm{~mm}$ Tris- $\mathrm{HCl}$ ( $\mathrm{pH} 8.0$ ), $5 \mathrm{~mm} \mathrm{MgCl} 2,5 \mathrm{~mm}$ mercaptoethanol, $4.6 \mu \mathrm{M}$ FPP and $0.5 \mu \mathrm{M} \mathrm{GecA}$ in a total volume of $2.5 \mathrm{ml}$. The reaction mixture, overlaid with $n$-hexane $(1 \mathrm{ml})$, was incubated at $30^{\circ} \mathrm{C}$ for $4 \mathrm{~h}$. The reaction was terminated by the addition of $0.5 \mathrm{M}$ EDTA $(\mathrm{pH} 8.0,200 \mu \mathrm{l})$ followed by immediate vortexing for $30 \mathrm{~s}$. The product(s) was extracted with $n$-hexane and subjected to GC-MS analysis. For determination of the optimum conditions for the GecA reaction, an in vitro assay was performed as follows. The standard reaction mixture contained $50 \mathrm{~mm}$ 4-(2-hydroxyethyl)-1-piperazine ethanesulfonic acid (HEPES) ( $\mathrm{pH} 8.0$ ), $1 \mathrm{~mm} \mathrm{MgCl}, 5 \mathrm{~mm}$ mercaptoethanol, $2.3 \mu \mathrm{M}$ FPP and $29 \mathrm{~nm} \mathrm{GecA}$ in a total volume of $2.5 \mathrm{ml}$. The reaction mixture, overlaid with $n$-hexane $(1 \mathrm{ml})$, was incubated at $35^{\circ} \mathrm{C}$ for $20 \mathrm{~min}$. The data were obtained from three independent experiments.

\section{Kinetic analysis}

The reaction mixture $\left(2.5 \mathrm{ml}\right.$ ) contained $50 \mathrm{~mm}$ HEPES (pH 8.0), $1 \mathrm{~mm} \mathrm{MgCl}_{2}$, $5 \mathrm{~mm}$ mercaptoethanol, FPP $(0.09$ to $4.6 \mu \mathrm{m})$ and $29 \mathrm{~nm}$ GecA. Each reaction mixture was incubated at $35^{\circ} \mathrm{C}$ for $3 \mathrm{~min}$. Each reaction was terminated by the addition of $0.5 \mathrm{M}$ EDTA ( $\mathrm{pH}$ 8.0, $200 \mu \mathrm{l}$ ) followed by immediate vortexing for $30 \mathrm{~s}$. The product was extracted with $n$-hexane and subjected to GC-MS analysis. We confirmed that the product formation was linear throughout this period. Steady-state parameters were determined by fitting the curve to $v=V_{\max }[\mathrm{S}] /\left(K_{\mathrm{m}}+[\mathrm{S}]\right)$, where $V_{\max }$ is the maximum reaction rate, $v$ is the initial velocity of formation of $(+)$-epicubenol by GecA and $S$ is the concentration of FPP. The data were obtained from three independent experiments.

\section{Gene disruption of gecA in $S$. griseus}

A 1.63-kbp DNA fragment, which contained a 1.48-kbp region upstream from the gecA start codon and a 150-bp region downstream from the start codon, was amplified by PCR using primer III, (5'-GCCAAGCTTATCCCGAAGGACTCCTTGTAGAAG-3' (HindIII site italicized) and primer IV, 5'-CGCA AGCTTATCGGCTTGTTCGAATCGGTTCCTC- $3^{\prime}$ (HindIII site italicized). Chromosomal DNA of S. griseus was used as a template for the PCR amplification. The PCR product was digested with HindIII and cloned into the HindIII site of pUC19, resulting in pUC-AgecAN. Similarly, a $1.48-\mathrm{kbp}$ DNA fragment, which contained a 627-bp region upstream from the gecA stop codon and a 850-bp region downstream from the stop codon, was amplified by PCR using primer $\mathrm{V}, 5^{\prime}$-TGGGGTACCGTCACCTACCTCACGACCGCCAC GACCTG-3' (KpnI site italicized) and primer VI, 5'-GCGGAATTCTTACG GGCTGCGGCACGCGGTGGTCGTCGAGTC- $3^{\prime}$ (EcoRI site italicized). The PCR product was digested with $K p n \mathrm{I}$ and EcoRI and cloned between the KpnI and EcoRI sites of pUC19, resulting in pUC- $\Delta$ gecAC. The kanamycin resistance gene (aphII) from Tn $5^{34}$ was inserted between the HindIII and KpnI sites of the pUC19- $\triangle g e c A C$, resulting in pUC19- $\Delta g e c A C K$. The HindIII fragment excised from the pUC19- $\Delta$ gecAN was cloned in the HindIII site of pUC19$\triangle$ gecACK, resulting in pUC19- $\Delta$ gecA, in which the middle of gecA sequence (encoding Phe-51 to Leu-150) was replaced by the aphII sequence. pUC19$\Delta g e c A$ was linearized with $\mathrm{DraI}$ and introduced by protoplast transformation into S. griseus IFO13350. Kanamycin-resistant transformants were selected, and the gene replacement, as a result of double crossover, was confirmed by Southern hybridization.

\section{Examination of terpenoid production in $S$. griseus}

The wild-type strain $S$. griseus, mutant $\triangle$ gecA::aphII and mutant $\triangle a d p A$ were grown in YMPD medium $(100 \mathrm{ml})$ at $30^{\circ} \mathrm{C}$ for $72 \mathrm{~h}$. Cells were harvested by centrifugation and resuspended in $50 \mathrm{~mm}$ Tris- $\mathrm{HCl}(\mathrm{pH} 8.0,20 \mathrm{ml})$, then disrupted by sonication. Low-molecular-weight molecules were then extracted with ethyl acetate from the crude cell lysate. The organic layer was dried with $\mathrm{Na}_{2} \mathrm{SO}_{4}$ and then analyzed by GC-MS.

\section{S1 nuclease mapping}

Total RNA was isolated with Isogen (Nippon Gene, Tokyo, Japan) from cells grown at $30{ }^{\circ} \mathrm{C}$ in YMPD liquid medium, as described previously. ${ }^{35} \mathrm{~S} 1$ nuclease mapping was performed by a method described by Bibb et al. ${ }^{36}$ and Kelemen et al. ${ }^{37}$ Hybridization probes were prepared by PCR with a pair of 
${ }^{32} \mathrm{P}$-labeled and unlabeled primers. $h r d B$ encoding a principal sigma factor of RNA polymerase was used to check the purity and amount of RNA used, as described previously. ${ }^{29}$ Primers used in S1 nuclease mapping are listed in Supplementary Table 1.

\section{RESULTS}

Identification of a terpenoid produced by the gecA overexpression strain

To analyze in vivo function of GecA, a gecA-overexpressing S. lividans strain was constructed. Low-molecular-weight molecules were extracted from the recombinant strain (or the negative control strain containing the empty vector pHSA81) using ethyl acetate. When the sample was analyzed by GC-MS, major product $\mathbf{1}$ was detected (Figure 2a). The mass spectrum of product 1 had peaks at $\mathrm{m} / \mathrm{z} 204$ and 207, corresponding to fragment ions of dehydrated $\left(\mathrm{C}_{15} \mathrm{H}_{26} \mathrm{O}-\right.$ $\left.\mathrm{H}_{2} \mathrm{O}\right)$ and demethylated $\left(\mathrm{C}_{15} \mathrm{H}_{26} \mathrm{O}-\mathrm{CH}_{3}\right)$ forms, respectively, of a sesquiterpene alcohol (Figure 2b). Product 1 was purified by $\mathrm{SiO}_{2}$ column chromatography, yielding $9.2 \mathrm{mg}$ of 1 from the 4-1 culture. The ${ }^{1} \mathrm{H}$ NMR spectrum displayed signals arising from one fully substituted methyl group $(\delta 1.54[3 \mathrm{H}, \mathrm{s}])$, three doublet methyl groups $(\delta 0.78,0.83$ and 1.10 [each $3 \mathrm{H}, \mathrm{d}, J=7.0 \mathrm{~Hz}])$ and one olefinic proton $(\delta 5.39[1 \mathrm{H}, \mathrm{bs}])$, while 12 remaining protons appeared in the $\delta 0.98-2.04$ region. The ${ }^{13} \mathrm{C}$ NMR spectrum showed 15 carbon resonances; one $\mathrm{C}-\mathrm{H}$ olefinic carbon $(\delta$ 122.5) and one
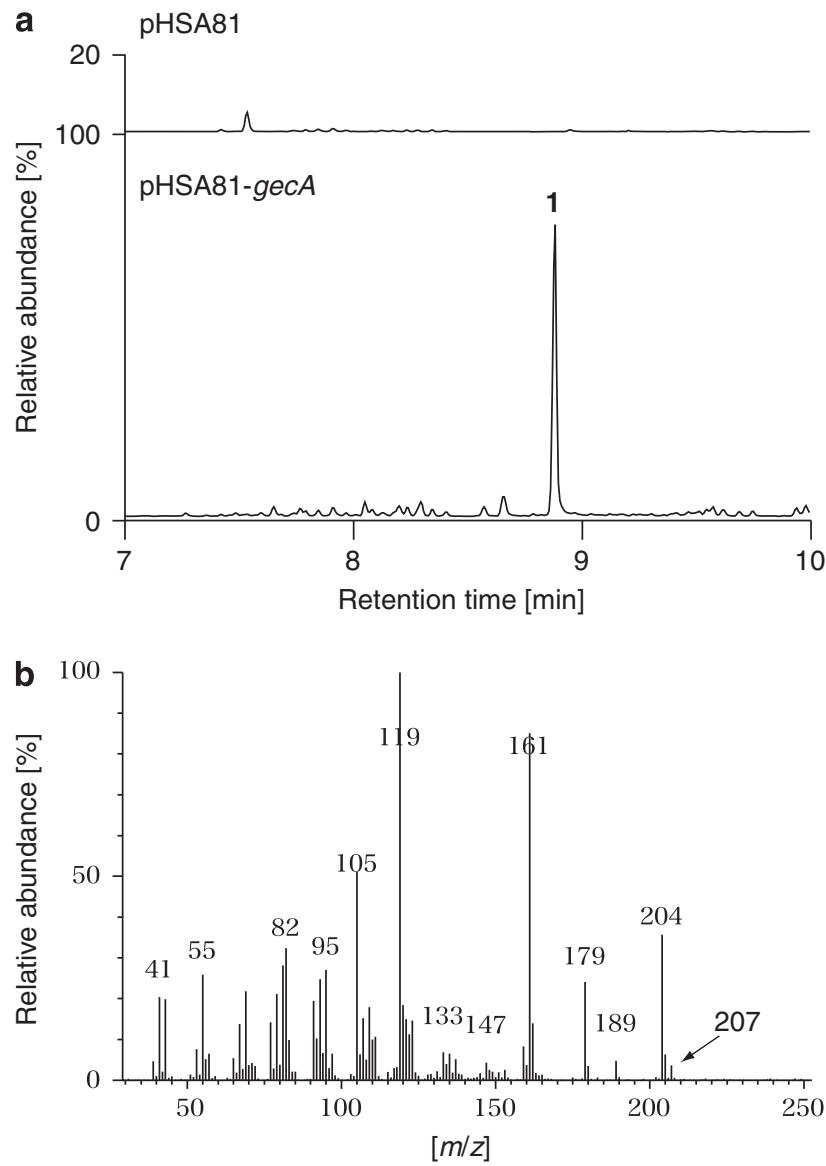

Figure 2 GC-MS analysis of extracts from crude cell lysate of recombinant S. lividans strains. (a) GC-MS chromatograms of extracts from crude cell lysate of $S$. lividans strains harboring empty vector pHSA81 and pHSA81gecA. (b) Mass spectrum of 1 . fully substituted olefinic carbon $(\delta$ 133.9). By two-dimensional NMR spectroscopic analyses, including COSY, HMQC, HMBC and NOESY experiments (Supplementary Figure S1), we determined the structure and relative stereochemistry of $\mathbf{1}$ the bicyclic sesquiterpene alcohol, epicubenol. The NMR spectra and signal assignments of $\mathbf{1}$ agreed completely with those previously reported for $(+)$-epicubenol. ${ }^{22}$ Finally, the absolute configuration of 1 was determined as $(+)$ by comparing the specific rotation value of $\mathbf{1}\left([\alpha]_{D}+111.6\right)$ with that of $(+)$-epicubenol from $H$. planus $\left([\alpha]_{D}+106.5\right) \cdot{ }^{20}$

\section{In vitro analysis of the GecA reaction}

GecA was produced as an N-terminal His-tagged protein $\left(\mathrm{MNHKVH}_{6} \mathrm{IEGRH}_{\mathrm{H}} \mathrm{GecA}\right)$ in E. coli. The recombinant GecA was purified by nickel-nitrilotriacetic acid affinity chromatography to give a single major protein band of $43 \mathrm{kDa}$ on SDS-PAGE (Supplementary Figure S2). Incubation of the recombinant GecA protein with FPP yielded $(+)$-epicubenol $(\mathbf{1})$ as a major product, as determined by GC-MS analysis (Figure 3a). In addition to 1 , at least three minor products were detected in the in vitro GecA reaction (Figure 3a). A minor product (at $8.6 \mathrm{~mm}$ ), 5, was identified as germacradien-4-ol by comparison of the retention time and mass fragmentation pattern with those of the authentic sample (Figure $3 \mathrm{a}$ and $\mathrm{b}$ ). Minor products 6 (at $8.3 \mathrm{~mm}$ ) and 7 (at $7.8 \mathrm{~mm}$ ) were predicted to be cubenene (see Figure 1 for structure) and $\beta$-gurjunene (see Figure $3 c$ for structure), respectively, by comparison of the mass spectrum with a GC-MS database (National Institute of Standards and Technology Libraries, NIST08) (Supplementary Figure S3). Although the mechanism of the reaction catalyzed by the bacterial $(+)$-epicubenol synthase from Streptomyces sp. LL-B7 has previously been characterized, no kinetic analysis of the enzyme was reported. We therefore analyzed the kinetics of GecA. First, the optimum $\mathrm{pH}$ and temperature of the GecA reaction were examined. The optimum $\mathrm{pH}$ for $(+)$-epicubenol synthesis at $35^{\circ} \mathrm{C}$ was found to be 8.0 (Figure 4a). The optimum temperature for $(+)$-epicubenol synthesis at $\mathrm{pH} 8.0$ was found to be $35^{\circ} \mathrm{C}$ (Figure $4 \mathrm{~b}$ ). The $K_{\mathrm{m}}$ value for FPP and the $k_{\text {cat }}$ value for $(+)$-epicubenol formation under these optimized conditions $(\mathrm{pH} 8.0$ and $35^{\circ} \mathrm{C}$ ), in the presence of $1 \mathrm{~mm} \mathrm{MgCl}_{2}$ (see below), were measured as $254 \pm 7.1 \mathrm{nM}$ and $0.026 \pm 0.001 \mathrm{~s}^{-1}$, respectively (Figure $4 \mathrm{c})$. The $k_{\mathrm{cat}} / K_{\mathrm{m}}$ value $\left(0.10 \mathrm{~s}^{-1} \mu \mathrm{M}^{-1}\right)$ was broadly comparable with those reported for known bacterial SCs. ${ }^{7,9,10}$ GecA requires divalent metal ions for its activity. $\mathrm{Mg}^{2+}$ was the most effective among the divalent metal ions examined, and more than $1 \mathrm{~mm} \mathrm{MgCl}_{2}$ was required for maximum activity (data not shown). In the presence of $\mathrm{Mn}^{2+}\left(1 \mathrm{mM} \mathrm{MnCl}_{2}\right)$, GecA showed only $12 \%$ activity, relative to the activity in the presence of $\mathrm{Mg}^{2+}$. In contrast, no activity was observed for $\mathrm{Fe}^{2+}, \mathrm{Co}^{2+}, \mathrm{Zn}^{2+}, \mathrm{Ni}^{2+}$ or $\mathrm{Cu}^{2+}$ (each at $1 \mathrm{~mm}$ as chloride salt).

Production of $(+)$-epicubenol by wild-type $S$. griseus and an $\operatorname{adp} A$-deleted mutant but not by a gecA-knockout mutant

To examine the production of $(+)$-epicubenol in $S$. griseus, we analyzed the extracts of the crude cell lysate of the wild-type and gecAdisrupted strains by GC-MS (Figure 5). Because a fragment ion peak with $\mathrm{m} / \mathrm{z} 119$ was detected for $(+)$-epicubenol (1) (Figure 2b), ${ }^{20}$ production of 1 was assessed by monitoring the fragment ion peak with $\mathrm{m} / z 119$ in the GC-MS analysis (Figure 5). A peak at $8.9 \mathrm{~min}$ was detected in the selected ion chromatogram of the wild-type sample, but not in the $\Delta g e c A:: a p h I I$ sample. This peak was shown to come from $(+)$-epicubenol by comparison with the authentic sample (Figure 5). From this result, we concluded that gecA is not a cryptic gene and is responsible for $(+)$-epicubenol production in S. griseus. 

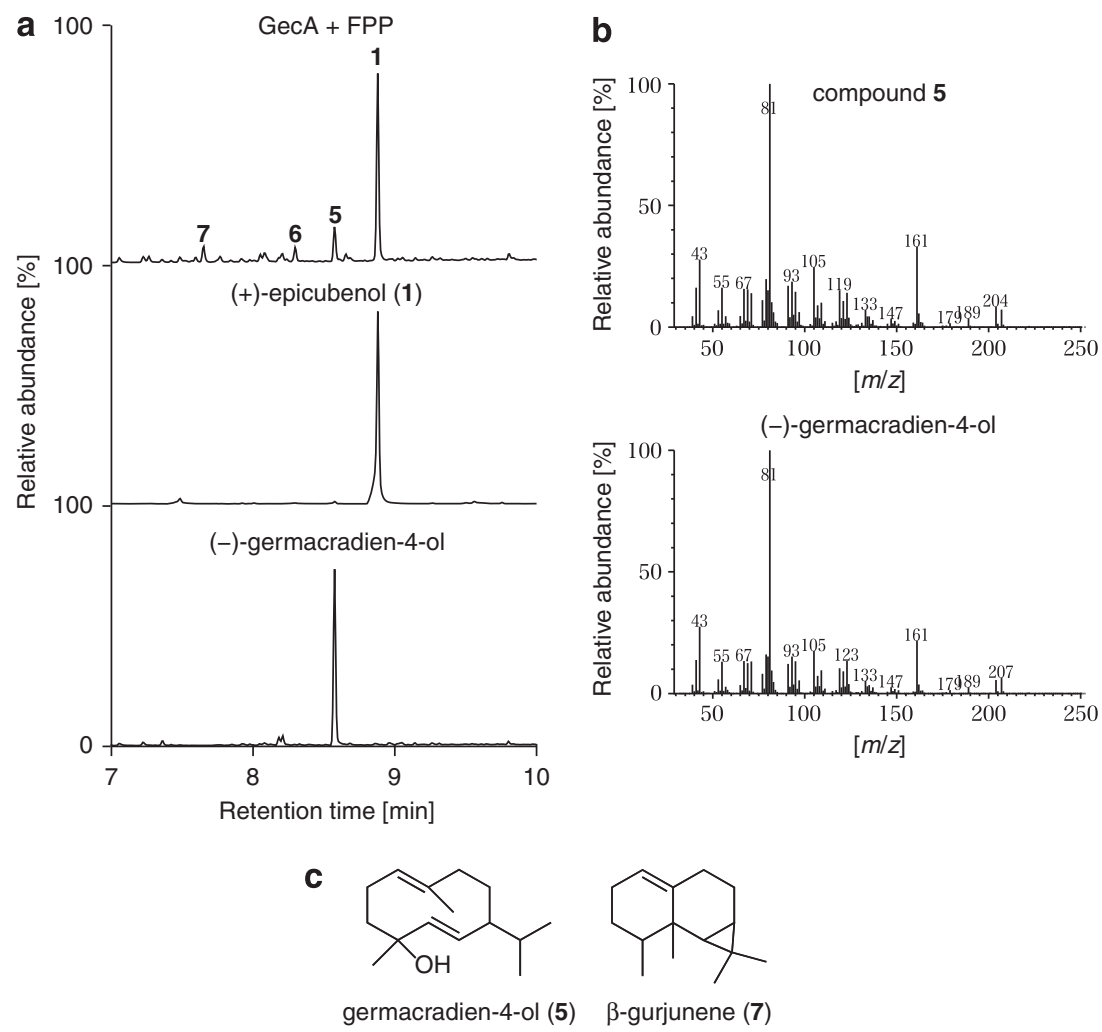

Figure 3 In vitro reaction of recombinant GecA protein. (a) GC-MS chromatograms of products generated by GecA with FPP, ( + )-epicubenol and (-)-germacradien-4-ol. (b) Mass spectrum of 5 and (-)-germacradien-4-ol. (c) Structures of germacradiene-4-ol (5) and $\beta$-gurjunene (7).

Because gecA did not appear to be a direct target of AdpA, ${ }^{31}$ a master regulator of secondary metabolism and morphological differentiation in S. griseus, we also analyzed the extracts of the crude cell lysate of an adpA-deleted $(\triangle a d p A)$ mutant by GC-MS (Figure 5). As expected, the production of 1 by the $\triangle a d p A$ mutant could be detected, confirming that $(+)$-epicubenol production is not controlled by A-factor in S. griseus. Interestingly, the $\triangle a d p A$ mutant produced more $(+)$ epicubenol than the wild-type strain.

\section{Transcription profiles of $g e c A$ and $g c o A$ in the wild-type and adpA-deleted strains}

As described above, the $\triangle a d p A$ strain produced more $(+)$-epicubenol than the wild-type strain. In contrast, $(+)$-caryolan-1-ol, which is synthesized by another single-domain SC, GcoA, is produced in the wild-type $S$. griseus strain, but not in the $\triangle a d p A$ mutant. ${ }^{12}$ Therefore, we analyzed expression profiles of $g e c A$ and $g c o A$ in the wild-type and $\triangle a d p A$ strains by $\mathrm{S} 1$ nuclease mapping. RNAs were extracted from each strain grown for 12 (mid log phase), 21 (late log phase) and $32 \mathrm{~h}$ (stationary phase) in liquid YMPD. gecA was transcribed throughout growth; the transcription was slightly more active in mid log phase (Figure 6). No significant difference in the amount of gecA transcript was observed between the wild-type and $\triangle a d p A$ strains (Figure 6), indicating that the observed difference in the productivity of $(+)$ epicubenol could not be ascribed to differences in the transcriptional level of gecA. Unexpectedly, transcription of $g c o A$ was detected in the $\triangle a d p A$ mutant strain, similar to the wild-type strain, almost constantly throughout growth (Figure 6). This result apparently contradicted the observation that the $\triangle a d p A$ strain did not produce $(+)$-caryolan-1-ol. ${ }^{12}$ Presence of a TTA codon in $g c o A$ and regulation of the tRNA-UUA gene by AdpA could explain this apparent contradiction (see Discussion).

\section{DISCUSSION}

In this study, we demonstrated that GecA is a SC involved in $(+)$ epicubenol synthesis by $S$. griseus. (+)-Epicubenol synthases have been previously purified and characterized from the liverwort H. planus and Streptomyces sp. LL-B7. However, no (+)-epicubenol synthase gene has been cloned to date, and gecA is thus the first example of an $(+)$-epicubenol synthase-encoding gene. The identification of GecA as an $(+)$-epicubenol synthase enabled a phylogenic comparison of the $(+)$-epicubenol synthase to be made with other known SCs and SC homologs. Six close homologs of GecA were found in other Streptomyces species; S. griseus XylebKG-1 (SACT1_6398, 100\% amino-acid identity with GecA), Streptomyces roseosporus NRRL 11379 (SrosN1_010100003716, 95\%), Streptomyces sp. W007 (SPW_4380, 95\%), S. roseosporus NRRL 15998 (SSGG_00557, 94\%), Streptomyces sp. SirexAA-E (SACTE_0873, $84 \%$ ) and Streptomyces flavogriseus ATCC 33331 (Sfla_5399, 81\%). These GecA homologs are presumed to be $(+)$-epicubenol synthases. Very recently, Citron et al. ${ }^{38}$ reported that S. griseus IFO13350 (NBRC13350), S. roseosporus NRRL15998 and S. flavogriseus ATCC33331 produce epicubenol. SSGG_00557 and Sfla_5399 therefore should be responsible for the production of epicubenol. Together with the observation that three Streptomyces species, Streptomyces sp. LL-B7, sp. LL-B5a and sp. LL-100-1 (Eren), produce $(+)$-epicubenol, ${ }^{19}$ this sesquiterpene alcohol appears to be a relatively common secondary metabolite in the genus Streptomyces. 

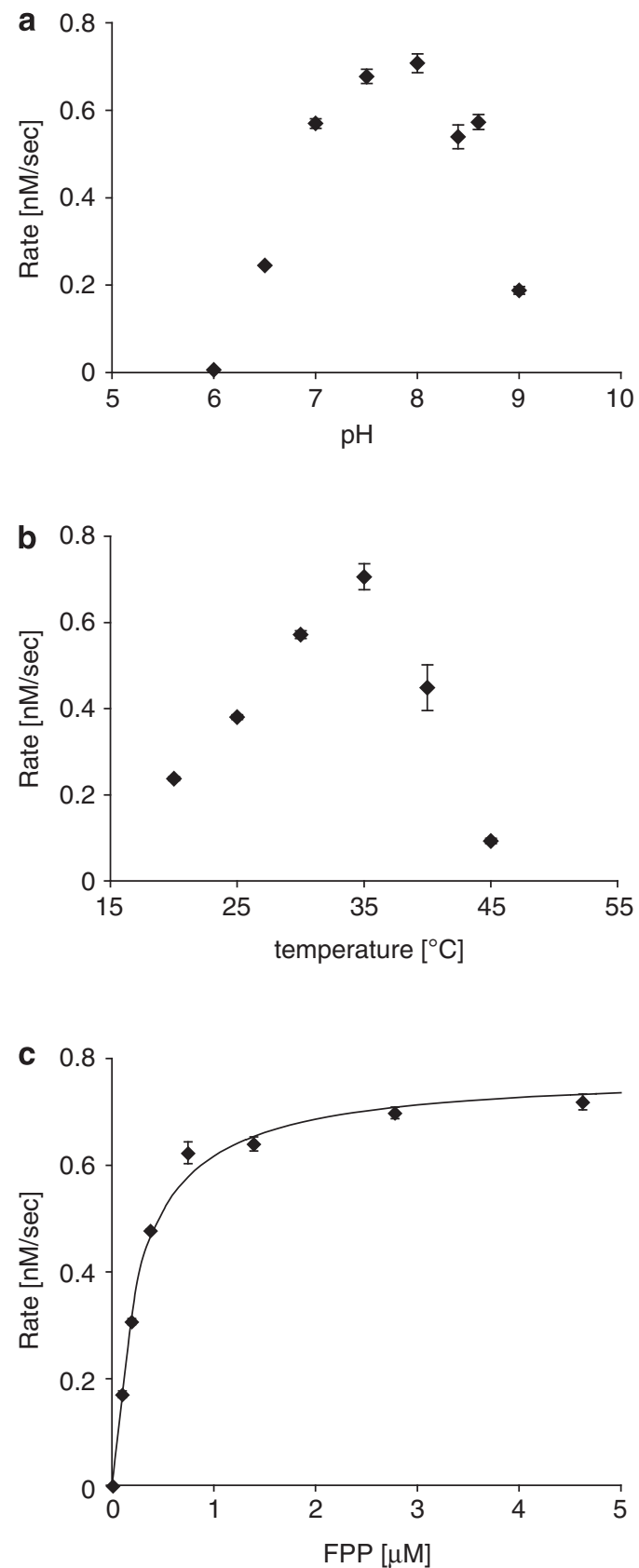

Figure 4 Analysis of the optimum $\mathrm{pH}$ and temperature for the GecA reaction and kinetic analysis of GecA. (a) Recombinant GecA was incubated with $\mathrm{FPP}$ at $35^{\circ} \mathrm{C}$ at various $\mathrm{pHs} ; 50 \mathrm{~mm}$ 4-morpholineethanesulfonic acid (MES) buffer ( $\mathrm{pH}$ 6.0-6.5), $50 \mathrm{~mm}$ HEPES buffer ( $\mathrm{pH} 7.0-8.4$ ) and $50 \mathrm{~mm} N$-cyclohexyl-2-aminoethanesulfonic acid (CHES) buffer (pH 8.6-9.0) were used. (b) Recombinant GecA was incubated with FPP in $50 \mathrm{~mm}$ HEPES buffer $(\mathrm{pH} 8.0)$ at various temperatures $\left(20-45^{\circ} \mathrm{C}\right)$. (c) The rate-FPP concentration profile for $(+)$-epicubenol synthesis from FPP are shown. The data were obtained from three independent experiments.

GecA catalyzes the conversion of FPP into (+)-epicubenol, probably with the same reaction mechanism as has been previously elucidated for the $(+)$-epicubenol synthase from Streptomyces sp. LL-B7 (Figure 1). Three minor products, germacradien-4-ol and the putative products cubenene and $\beta$-gurjunene, were detected in the in vitro GecA reaction. Germacradien-4-ol and $\beta$-gurjunene are

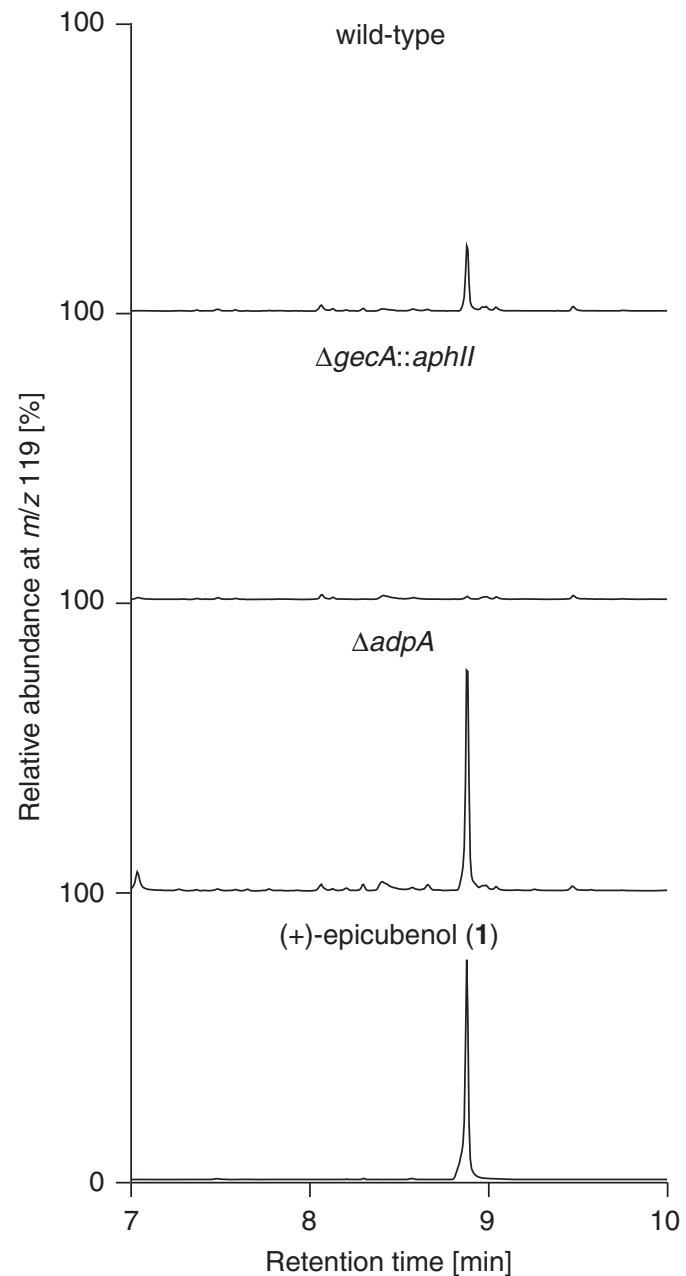

Figure 5 GC-MS analysis of terpenoids produced by $S$. griseus. GC-MS chromatograms of the extracts of wild-type $S$. griseus and mutants $\Delta g e c A:$ aphll and $\triangle a d p A$, and (+)-epicubenol.

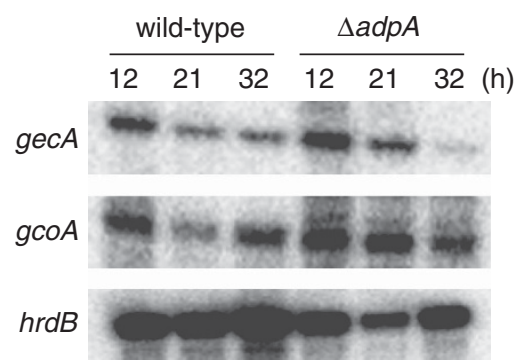

Figure 6 Time course of transcription of gecA and $g c 0 A$, as determined by low-resolution S1 nuclease mapping. RNA was prepared from the wild-type and $\triangle a d p A$ strains grown at $30^{\circ} \mathrm{C}$ in liquid YMPD for the times indicated above the panels. The $h r d B$ gene encoding a principal sigma factor was used as an internal control.

predicted to be generated from the $(E, E)$-germacradienyl cation formed by ionization-initiated cyclization of FPP (Supplementary Figure S4). Thus, we postulate that GecA produces small amount of $(E, E)$-germacradienyl cation, in addition to $(Z, E)$-germacradienyl cation (2), at least in the in vitro reaction. The multiproduct terpene synthase from Medicago truncatula generates both $(E, E)$ 
and $(Z, E)$-germacradienyl cations. ${ }^{39}$ However, it is also possible that germacradien-4-ol is generated from a reaction intermediate, $(Z$, E)-germacradienyl cation (2), of the $(+)$-epicubenol synthesis (Figure 1). ${ }^{40}$ Cubenene can also be generated from another reaction intermediate, bicyclic cation (4) (Figure 1). The SC involved in (+)epicubenol biosynthesis in $H$. planus also produces $(+)$-cubenene; an in vitro enzyme reaction yielded both $(+)$-epicubenol and $(+)$ cubenene with a molar ratio of $1: 3,{ }^{21}$ indicating that the major product is $(+)$-cubenene. In contrast, GecA produces only a small amount of cubenene (Figure 3a). In the synthesis of $(+)$-epicubenol, the bicyclic cation (4) is quenched by the addition of a water molecule ('path a' in Figure 1). ${ }^{25}$ Cane et al. ${ }^{25}$ speculated that the same water molecule might serve as the Lewis base in the deprotonation reaction ('path b' in Figure 1) that would lead to the formation of cubenene. In the reaction pocket of the $H$. planus $(+)$-cubenene/ $(+)$-epicubenol synthase, deprotonation of the bicyclic cation (4) may occur much more easily compared with GecA. Moreover, it is interesting that the reactions catalyzed by $(+)$-T-muurolol synthase are partially identical to those catalyzed by GecA, although $(+)$-T-muurolol synthase from $S$. clavuligerus shows a low amino-acid sequence similarity (25\% identity) to GecA. In (+)-T-muurolol synthesis, the cis-fused muurolyl cation (3) is produced, and this intermediate is quenched by the addition of a water molecule (Figure 1). ${ }^{11}$

We reported that transcription of $g c o A$ was enhanced by exogenous A-factor in an A-factor-deficient mutant, as determined by DNA microarray analysis (fold change 4.1-5.5; $P<0.01 ; 4-12 \mathrm{~h}$ after A-factor addition). ${ }^{31}$ Our recent transcriptome analysis also showed that $g c o A$ was downregulated in the absence of adpA (fold change 3.8; $P=0.003) .{ }^{41}$ In contrast, our previous transcriptome analysis showed that $g c o A$ was slightly upregulated in the $\triangle a d p A$ strain compared with the wild-type strain (fold change $1.3 ; P=0.001$ ). ${ }^{26}$ In the present study, we found that the transcription profile of $g c o A$ was similar in the wild-type and $\triangle a d p A$ strains, as determined by S1 nuclease mapping. This result indicated that AdpA should not necessarily be required for the transcription of $g c o A$. The lack of $(+)$-caryolan-1-ol production by the $\triangle a d p A$ strain could be explained by the presence of a TTA codon (for Leu-6) in gcoA. The UUA leucine codon is very rare in Streptomyces, and bldA encodes the only tRNA species able to read the UUA codon efficiently. Recently, it was shown that AdpA bound to the upstream region of $b l d A$ and activated transcription of bldA. ${ }^{42}$ Thus, the TTA-containing $g c o A$ gene is presumably not translated efficiently in the $\triangle a d p A$ mutant and GcoA is not produced in the $\triangle a d p A$ mutant, which results in no production of $(+)$-caryolan-1-ol.

Genome sequencing has revealed that S. griseus IFO13350 has more than 34 (putative) biosynthesis gene clusters (or genes) thought to be involved in secondary metabolite production. ${ }^{26}$ However, the products of most of these clusters are yet to be identified. The secondary metabolites of S. griseus IFO13350 that have been identified to date are streptomycin, ${ }^{43}$ grixazone, ${ }^{44}$ 1,4,6,7,9,12hexahydroxyperylene-3,10-quinone, ${ }^{45}$ alkylquinones, ${ }^{46}$ a carotenoid, ${ }^{47}$ geosmin, ${ }^{48}$ 2-methylisoborneol, ${ }^{48}$ and $(+)$-caryolan-1-ol. ${ }^{12}$ The production of streptomycin, grixazone, hexahydroxyperylene-3,10quinone and $(+)$-caryolan-1-ol has been shown to be under the control of the A-factor regulatory cascade, either directly or indirectly. In contrast, the production of alkylquinones, geosmin, and 2-methylisoborneol does not appear to be controlled by A-factor. The production of the carotenoid is cryptic under normal culture conditions. In this study, we succeeded in identifying $(+)$-epicubenol as a secondary metabolite produced by $S$. griseus and confirmed that its production is not controlled by A-factor. In contrast, production of $(+)$-caryolan-1-ol is controlled by A-factor through the translational regulation of $g c o A$ by the BldA tRNA, which is produced in an AdpA-dependent manner. Thus, $S$. griseus produces two sesquiterpene alcohols using single-domain SCs; $(+)$-caryolan-1-ol, whose production is A-factor dependent, and $(+)$-epicubenol, whose production is independent of A-factor. This finding provides new insight into the regulation of secondary metabolism by A-factor in S. griseus. It should be noted that neither the gcoA mutant nor the gecA mutant showed any phenotypic changes with respect to growth or morphological differentiation.

Very recently, the production of a great variety of volatile terpenoids from 35 strains, including 31 actinomycetes and four strains from other taxa, was reported. ${ }^{38}$ This result, together with several genome mining studies for SC homologs by our group ${ }^{12-15}$ and others, ${ }^{2-11}$ indicates that terpenoids are significantly more widely distributed in nature than was previously appreciated, being widespread among not only eukaryotes, but also bacteria.

\section{ACKNOWLEDGEMENTS}

This work was supported in part by the Targeted Proteins Research Program (TPRP) of the Ministry of Education, Culture, Sports, Science and Technology of Japan, and a Funding Program for Next Generation World-Leading Researchers from the Bureau of Science, Technology, and Innovation Policy, Cabinet Office, Government of Japan.

1 Chappell, J. Biochemistry and molecular biology of the isoprenoid biosynthetic pathway in plants. Annu. Rev. Plant Physiol. Plant Mol. Biol. 46, 521-547 (1995).

2 Cane, D. E. \& Watt, R. M. Expression and mechanistic analysis of a germacradienol synthase from Streptomyces coelicolor implicated in geosmin biosynthesis. Proc. Natl Acad. Sci. USA 100, 1547-1551 (2003).

3 Cane, D. E., He, X., Kobayashi, S., Ōmura, S. \& Ikeda, H. Geosmin biosynthesis in Streptomyces avermitilis. J. Antibiot. 59, 471-479 (2006).

4 Ghimire, G. P., Oh, T. -J., Lee, H. C., Kim, B. -G. \& Sohng, J. K. Cloning and functional characterization of the germacradienol synthase (spterp13) from Streptomyces peucetius ATCC 27952. J. Microbiol. Biotechnol. 18, 1216-1220 (2008).

5 Giglio, S., Jiang, J., Saint, C. P. Cane, D. E. \& Monis, P. T. Isolation and characterization of the gene associated with geosmin production in cyanobacteria. Environ. Sci. Technol. 42, 8027-8032 (2008).

6 Tetzlaff, C. N., You, Z., Cane, D. E., Takamatsu, S., Ōmura, S. \& Ikeda, H. A gene cluster for biosynthesis of the sesquiterpenoid antibiotic pentalenolactone in Streptomyces avermitilis. Biochemistry 45, 6179-6186 (2006)

7 Lin, X., Hopson, R. \& Cane, D. E. Genome mining in Streptomyces coelicolor: Molecular cloning and characterization of a new sesquiterpene synthase. J. Am. Chem. Soc. 128, 6022-6023 (2006).

8 Takamatsu, S., Lin, X., Nara, A., Komatsu, M., Cane, D. E. \& Ikeda, H. Characterization of a silent sesquiterpenoid biosynthetic pathway in Streptomyces avermitilis controlling epi-isozizaene albaflavenone biosynthesis and isolation of a new oxidized epi-isozizaene metabolite. Microb. Biotechnol. 4, 184-191 (2011).

9 Chou, W. K. W., Fanizza, I., Uchiyama, T., Komatsu, M., Ikeda, H. \& Cane, D. E. Genome mining in Streptomyces avermitilis: cloning and characterization of SAV_76, the synthase for a new sesquiterpene, avermitilol. J. Am. Chem. Soc. 132, 8850-8851 (2010).

10 Agger, S. A., Lopez-Gallego, F., Hoye, T. R. \& Schmidt-Dannert, C. Identification of sesquiterpene synthases from Nostoc punctiforme PCC 73102 and Nostoc sp. strain PCC 7120. J. Bacteriol. 190, 6084-6096 (2008).

$11 \mathrm{Hu}$, Y., Chou, W. K. W., Hopson, R. \& Cane, D.E. Genome mining in Streptomyces clavuligerus: expression and biochemical characterization of two new cryptic sesquiterpene synthases. Chem. Biol. 18, 32-37 (2011).

12 Nakano, C., Horinouchi, S. \& Ohnishi, Y. Characterization of a novel sesquiterpene cyclase involved in (+)-caryolan-1-ol biosynthesis in Streptomyces griseus. J. Biol. Chem. 286, 27980-27987 (2011).

13 Nakano, C., Kim, H. -K. \& Ohnishi, Y. Identification of the first bacterial monoterpene cyclase, a 1,8-cineole synthase, that catalyzes the direct conversion of geranyl diphosphate. Chembiochem 12, 1988-1991 (2011).

14 Nakano, C., Kim, H. -K. \& Ohnishi, Y. Identification and characterization of the linalool/ nerolidol synthase from Streptomyces clavuligerus. Chembiochem 12, 2403-2407 (2011).

15 Nakano, C., Kudo, F. Eguchi, T. \& Ohnishi, Y. Genome mining reveals two novel bacterial sesquiterpene cyclases: (-)-germacradien-4-ol and (-)-epi- $\alpha$ bisabolol synthases from Streptomyces citricolor. Chembiochem 12, 2271-2275 (2011).

16 Cane, D. E. \& Ikeda, H. Exploration and mining of the bacterial terpenome. Acc. Chem. Res. 45, 463-472 (2012). 
17 Connolly, J. D., Phillips, W. R. \& Huneck, S. (+)-Ent-epicubenol from the liverwort Scapania undulata. Phytochemistry 21, 233-234 (1982).

18 Nabeta, K., Katayama, K., Nakagawara, S. \& Katoh, K. Sesquiterpenes of cadinane type from cultured cells of the liverwort, Heteroscyphus planus. Phytochemistry 32, 117-122 (1993).

19 Gerber, N. N. Sesquiterpenoids from actinomycetes: cadin-4-ene-1-ol. Phytochemistry 10, 185-189 (1971).

20 Nabeta, K., Kigure, K., Fujita, M., Nagoya, T., Ishikawa, T., Okuyama, H. \& Takasawa, T. Biosynthesis of $(+)$-cubenene and $(+)$-epicubenol by cell-free extracts of cultured cells of Heteroscyphus planus and cyclization of $\left[{ }^{2} \mathrm{H}\right]$ farnesyl diphosphates. J. Chem. Soc. Perkin Trans. 1, 1935-1939 (1995).

21 Nabeta, K., Fujita, M., Komuro, K., Katayama, K. \& Takasawa, T. In vitro biosynthesis of cadinanes by cell-free extracts of cultured cells of Heteroscyphus planus. J. Chem. Soc. Perkin Trans. 1, 2065-2070 (1997).

22 Cane, D. E., Tandon, M. \& Prabhakaran, P. C. Epicubenol synthase and the enzymatic cyclization of farnesyl diphosphate. J. Am. Chem. Soc. 115, 8103-8106 (1993).

23 Cane, D. E. \& Tandon, M. Biosynthesis of (+)-epicubenol. Tetrahedron lett. 35, 5355 5358 (1994)

24 Cane, D. E. \& Tandon, M. Epicubenol synthase and the stereochemistry of the enzymatic cyclization of farnesyl and nerolidyl diphosphate. J. Am. Chem. Soc. 117, 5602-5603 (1995).

25 Cane, D. E. \& Ke, N. Epicubenol synthase. Origin of the oxygen atom of a bacterial sesquiterpene alcohol. Bioorg. Med. Chem. Lett. 10, 105-107 (2000).

26 Ohnishi, Y., Ishikawa, J., Hara, H., Suzuki, H., Ikenoya, M., Ikeda, H., Yamashita, A., Hattori, M. \& Horinouchi, S. Genome sequence of the streptomycin-producing microorganism Streptomyces griseus IFO 13350. J. Bacteriol. 190, 4050-4060 (2008).

27 Horinouchi, S. \& Beppu, T. Hormonal control by A-factor of morphological development and secondary metabolism in Streptomyces. Proc. Jpn. Acad. Ser. B 83, 277-295 (2007).

28 Kato, J., Funa, N., Watanabe, H., Ohnishi, Y. \& Horinouchi, S. Biosynthesis of $\gamma$-butyrolactone autoregulators that switch on secondary metabolism and morphological development in Streptomyces. Proc. Natl Acad. Sci. USA 104, 2378-2383 (2007).

29 Ohnishi, Y., Kameyama, S., Onaka, H. \& Horinouchi, S. The A-factor regulatory cascade leading to streptomycin biosynthesis in Streptomyces griseus: identification of a target gene of the A-factor receptor. Mol. Microbiol. 34, 102-111 (1999).

30 Ohnishi, Y., Yamazaki, H., Kato, J., Tomono, A. \& Horinouchi, S. AdpA, a central transcriptional regulator in the A-factor regulatory cascade that leads to morphological development and secondary metabolism in Streptomyces griseus. Biosci. Biotechnol. Biochem. 69, 431-439 (2005).

31 Hara, H., Ohnishi, Y. \& Horinouchi, S. DNA microarray analysis of global gene regulation by A-factor in Streptomyces griseus. Microbiology 155, 2197-2210 (2009).

32 Cane, D. E., Sohng, J. -K., Lamberson, C. R., Rudnicki, S. M., Wu, Z., Lloyd, M. D., Oliver, J. S. \& Hubbard, B. R. Pentalenene synthase. purification, molecular cloning, sequencing, and high-level expression in Escherichia coli of a terpenoid cyclase from Streptomyces UC5319. Biochemistry 33, 5846-5857 (1994).

33 Christianson, D. W. Structural biology and chemistry of the terpenoid cyclases. Chem. Rev. 106, 3412-3442 (2006)
34 Beck, E., Ludwig, G., Auerswald, E. A., Reiss, B. \& Schaller, H. Nucleotide sequence and extract localization of the neomycin phosphotransferase gene from transposon Tn5. Gene 19, 327-336 (1982).

35 Tezuka, T., Hara, H., Ohnishi, Y. \& Horinouchi, S. Identification and gene disruption of small noncoding RNAs in Streptomyces griseus. J. Bacteriol. 191, 4896-4904 (2009).

36 Bibb, M. J., Janssen, G. R. \& Ward, J. M. Cloning and analysis of the promoter region of the erythromycin resistance gene (ermE) of Streptomyces erythraeus. Gene 41, 357368 (1986).

37 Kelemen, G. H., Brian, P., Flärdh, K., Chamberlin, L, Chater, K. F. \& Buttner, M. J. Developmental regulation of transcription of whiE, a locus specifying the polyketide spore pigment in Streptomyces coelicolor A3(2). J. Bacteriol. 180, 2515-2521 (1998).

38 Citron, C. A., Gleitzmann, J., Laurenzano, G., Pukall, R. \& Dickschat, J. S. Terpenoids are widespread in actinomycetes: a correlation of secondary metabolism and genome data. Chembiochem 13, 202-214 (2012).

39 Garms, S., Köllner, T. G. \& Boland, W. A multiproduct terpene synthase from Medicago truncatula generates cadalane sesquiterpenes via two different mechanisms. J. Org. Chem. 75, 5590-5600 (2010).

40 Yoshikuni, Y., Martin, V. J. J., Ferrin, T. E. \& Keasling, J. D. Engineering cotton (+ )- $\delta-$ cadinene synthase to an altered function: germacrene D-4-ol synthase. Chem. Biol. 13, 91-98 (2006).

41 Higo, A., Hara, H., Horinouchi, S. \& Ohnishi, Y. Genome-wide distribution of AdpA, a global regulator for secondary metabolism and morphological differentiation in Streptomyces, revealed the extent and complexity of the AdpA regulatory network. DNA Res. 19, 259-274 (2012).

42 Higo, A., Horinouchi, S. \& Ohnishi, Y. Strict regulation of morphological differentiation and secondary metabolism by a positive feedback loop between two global regulators AdpA and BIdA in Streptomyces griseus. Mol. Microbiol. 81, 1607-1622 (2011)

43 Tomono, A., Tsai, Y., Yamazaki, H., Ohnishi, Y. \& Horinouchi, S. Transcriptional control by A-factor of strR, the pathway-specific transcriptional activator for streptomycin biosynthesis in Streptomyces griseus. J. Bacteriol. 187, 5595-5604 (2005).

44 Higashi, T., Iwasaki, Y., Ohnishi, Y. \& Horinouchi, S. A-factor and phosphate depletion signals are transmitted to the grixazone biosynthesis genes via the pathway-specific transcriptional activator GriR. J. Bacteriol. 189, 3515-3524 (2007).

45 Funa, N., Funabashi, M., Ohnishi, Y. \& Horinouchi, S. Biosynthesis of hexahydroxyperylenequinone melanin via oxidative aryl coupling by cytochrome P-450 in Streptomyces griseus. J. Bacteriol. 187, 8149-8155 (2005).

46 Funabashi, M., Funa, N. \& Horinouchi, S. Phenolic lipids synthesized by type III polyketide synthase confer penicillin resistance on Streptomyces griseus. J. Biol. Chem. 283, 13983-13991 (2008).

47 Lee, H. -S., Ohnishi, Y. \& Horinouchi, S. A $\sigma^{B}$-like factor responsible for carotenoid biosynthesis in Streptomyces griseus. J. Mol. Microbiol. Biotechnol. 3, 95-101 (2001).

48 Komatsu, M., Tsuda, M., Ōmura, S., Oikawa, H. \& Ikeda, H. Identification and functional analysis of genes controlling biosynthesis of 2-methylisoborneol. Proc. Natl Acad. Sci. USA 105, 7422-7427 (2008).

Supplementary Information accompanies the paper on The Journal of Antibiotics website (http://www.nature.com/ja) 